Article

\title{
Evaluation of Face Shields, Goggles and Safety Glasses as a Virus Transmission Control Measure to Protect the Wearer Against Cough Droplets
}

\author{
Samantha Hall *, Paul Johnson, Claire Bailey, Zoe Gould, Robert White and Brian Crook
}

HSE Science and Research Centre, Harpur Hill, Buxton, Derbyshire, SK17 9JN

* Correspondence: samantha.hall@hse.gov.uk 02030282185

\begin{abstract}
Face shields (also referred to as visors), goggles and safety glasses have been worn during the COVID-19 pandemic as one measure to control transmission of the virus. However, their effectiveness in controlling facial exposure to cough droplets is not well established and standard tests for evaluating eye protection for this application are limited. A method was developed to evaluate face shields, goggles and safety glasses as a control measure to protect the wearer against cough droplets. The method uses a semi-quantitative assessment of facial droplet deposition. A cough simulator was developed to generate droplets comparable to those from a human cough. The droplets consisted of a UV fluorescent marker (fluorescein) in water. Fourteen face shields, four pairs of goggles and one pair of safety glasses were evaluated by mounting them on two different sizes of breathing manikin head and challenging them with the simulated cough. The manikin head was positioned in seven orientations relative to the cough simulator to represent various potential occupational exposure scenarios, for example, a nurse standing over a patient. Droplet deposition in the eyes, nose and mouth regions were visualised following three 'coughs'. Face shields, goggles and safety glasses reduced, but did not eliminate exposure to the wearer from droplets such as those produced by a human cough. The level of protection differed based on the design of the personal protective equipment and the relative orientation of the wearer to the cough. For example, face shields and goggles offered the greatest protection when a cough challenge was face on or from above and the least protection when a cough challenge was from below. Face shields were also evaluated as source control to protect others from the wearer. Results suggested that if a coughing person wears a face shield, it can provide some protection from cough droplets to those standing directly in front of the wearer.
\end{abstract}

Keywords: face shield; goggles; safety glasses; cough; droplets; virus transmission; COVID-19; exposure

\section{Introduction}

Face shields (also referred to as visors), goggles and safety glasses have been worn during the COVID-19 pandemic as one measure to control transmission of the virus. There are a number of studies that advise the use of eye protection to prevent contamination from external droplets and aerosol (Napoli et al 2020, Scalinci and Battagliola 2020) and associate eye protection with a lower risk of infection (Chu et al 2020 and Singh et al 2021). 
A medical face mask is considered a medical device and its primary intended use is to protect others from infective agents emitted by the wearer (BSI, 2019). A particle filtering half mask (respirator) is considered personal protective equipment and is worn to protect the wearer against solid and liquid aerosols (BSI, 2009). A face covering is broadly defined as something which safely covers the nose and mouth and is not considered a medical device or PPE (MHRA, 2021). Face shields have many advantages when compared to other face masks or coverings. They are robust, more comfortable, make it less likely for the wearer to touch their face, ease breathing, can be disinfected, reused, and communication is easier than when wearing a face mask or covering ( $\mathrm{Li}$ et al 2020, Salimina et al 2021, Singh et al 2021 and Verma et al 2021). In public and occupational settings, individuals are wearing face shields in place of, or in conjunction with, respirators, medical face masks or face coverings.

Some studies have concluded that face shields alone do not provide an adequate level of protection to the wearer against an infectious aerosol and that they should be worn in conjunction with other Personal Protective Equipment (PPE) or a medical face mask (Lindsley et al 2014, Roberge 2016, Samaranayake et al 2020, Salimina et al 2021). Although the use of face shields can substantially reduce the short-term exposure of the wearer to larger infectious aerosol particles and reduce contamination of a face mask or respirator worn underneath, face shields are less effective against smaller particles, which can remain airborne for extended periods and can easily flow around a face shield to be inhaled (Lindsley et al 2014).

Some studies have considered the effectiveness of face shields as source control to protect others from the wearer (Anon et al 2020, Verma et al 2020, and Li et al 2021). Face shields were found to prevent the forward motion of the expelled droplets, deflecting them downwards with smaller droplets remaining suspended. Pan et al 2020 found droplets smaller than $0.7 \mu \mathrm{m}$ travelled around the face shield but the face shield exhibited overall protection efficiencies of $\sim 75 \%$ (as source control) and $\sim 25 \%$ (to wearer) for $5 \mu \mathrm{m}$ particles. Wendling et al 2021 found that for short, close range exposure scenarios, when the receiver alone wore a face shield rather than a medical face mask (type I), there was a significant reduction in the number of particles, even with small particle size emission $(\sim 0.3 \mu \mathrm{m})$.

Anon et al 2020 and Ko-Keeneya et al 2020 considered the effect of a modified face shield design on the protection it provided. Both studies found a modified design that was more enclosed around the base of the chin provided more effective protection. Anon et al 2020 found that when wearing a familiar single-use standard face shield design, a simulated cough from the wearer was scattered along the side and lower parts of the visor and spray was observed across a significant part of the wearer's shirt. Ko-Keeneya et al 2020 also considered the relative head orientation of the wearer to the source of the cough and determined that there is inadequate protection provided by face shields of the common design particularly when leaning over the coughing person, exposing areas of the face and neck.

Eye protectors are tested to the European standard BS EN 166:2002 (BSI 2002a) before being placed on the UK market. This standard considers the basic optical and non-optical requirements applicable to all eyeprotectors and lists particular and optional tests specific to particular applications. 
BS EN 166:2002 does not include a test to evaluate protection afforded to the eyes against biological hazards. However; it does reference Clause 12 of BS EN 168:2002 (BSI 2002b) as a method for testing against droplets and fluids. Here, the tests vary according to whether the eye protector is goggles (providing protection against droplets) or a face shield (providing protection against larger splashes of liquid). There is no standard test to determine the splash protection of safety glasses. Face shields are not challenged by splashes in the standard test. As a surrogate, the face shield is required to cover a rectangular region around the eyes of a standard head form. The area of coverage is assessed by directing a laser towards this region whilst rotating the head form in various orientations to ensure the face shield continually intercepts the light.

Since the start of the COVID-19 pandemic, there has been an increase in the research published in this field. As new evidence emerged, UK PPE guidance for workplaces and the public, published by a range of public bodies and industry, has needed to be regularly reviewed and updated. Early in the pandemic, it was broadly believed that the main transmission vectors did not include airborne virus, therefore control measures were designed to protect individuals from cough droplets when working in close contact and where social distancing was not possible. Evidence latterly confirmed that the virus could be spread via airborne transmission. One such example is the guidance in Great Britain for close contact services such as hairdressers and barbers, spas, beauty salons, tattoo and photoshoot studios (BEIS and DCMS 2021). In July 2021, it advised that workers should 'wear further protection' against the COVID-19 risk. The guidance specified that this should be a face shield to provide a barrier between the wearer and the client from respiratory droplets caused by sneezing, coughing or speaking and there was no requirement for the client or practitioner to wear any additional protection or source control such as a mask or face covering, when the practitioner was wearing a visor. This suggested that the face shield was being worn to protect the worker but may also provide some protection to the client in this instance. Throughout the pandemic, this guidance has had many iterations, including the requirement for the client to wear a face covering, the addition of face masks for the practitioner then the easing of restrictions meaning that face coverings were no longer required by law (BEIS and DCMS 2021).

Throughout the COVID-19 pandemic, decisions and guidance have been based on best available evidence which, particularly at the outset, has been limited. The Health and Safety Executive (HSE) is the market surveillance authority for PPE in Great Britain. This paper describes how HSE researchers developed a method to evaluate the protection afforded to the wearer of safety glasses, goggles and face shields against droplets in such a way that is appropriate and proportionate to the risks associated with COVID-19. The method builds on BS EN 168:2002 with fluorescence visualisation techniques. It uses a human cough simulator adapted from an existing design for influenza and a manikin head. The paper also describes the findings from the use of this method to evaluate a range of safety glasses, googles and face shields procured by the UK Government. The research findings have informed HSE decision making and the development of evidence-based guidance for the control of COVID-19 transmission in workplaces. 


\section{Methods}

A cough simulator was adapted from an existing design (Lindsley et al 2013) which was based on flow rate measurements of coughs from 47 human subjects with influenza (Lindsley et al 2010). The simulator used in this study had a 'drive cylinder' that ejected 4.2 litres of air from a 'lung cylinder' through a 'mouth' outlet (Figure 1). The flow rate against time profile matched the target profile presented by Lindsley et al (2010). The outlet was connected perpendicularly to a plastic pipe $(1.1 \mathrm{~m}$ length $\times 0.04$ $\mathrm{m}$ diameter). One end of the pipe was connected to a Badger 200 Airbrush (Badger Air-brush Co, USA) used to spray an aqueous solution of $0.1 \%$ fluorescein into the pipe. Both sides of the drive cylinder were pressurised before valves were opened simultaneously to depressurise the front end and initiate the cough. The simulated human cough was directed towards a breathing manikin head.

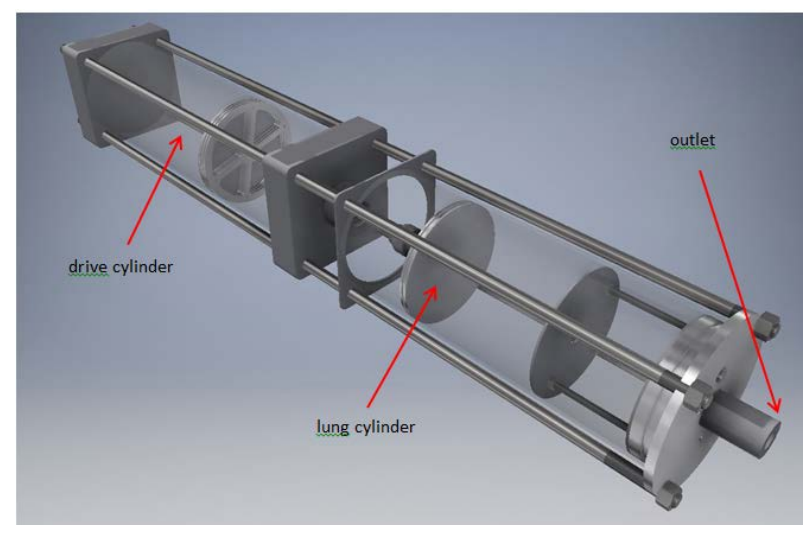

Figure 1. Design of the HSE cough simulator with the barrels transparent.

A bespoke test rig on which to mount the manikin heads was constructed following the design described in BS EN166 (BSI 2002b). The rig enabled the head to be tilted forward, backward and rotated side to side and locked in the required position. Tubing joined a connection beneath the neck of the head to a breathing machine (Inspec International Ltd) operating at a breathing rate of 20 breaths per minute with a 'lung capacity' of 2 litres.

Tests were conducted in a wooden test room (Supplementary Figure SF1) with internal dimensions of $3 \mathrm{~m} \mathrm{H} \times 4 \mathrm{~m} \mathrm{~W} \times 4 \mathrm{~m} \mathrm{D}$. The room was ventilated between tests to remove any fine particles from the air.

Fourteen face shield models were tested, representing a selection of models that were centrally procured and stockpiled by the United Kingdom Government for supply to the National Health Service (NHS) and wider health and social care. All models were evaluated within the context of European Commission recommendation 2020/403 to meet the increased demand for PPE during the COVID-19 pandemic (EU, 2020). Twelve of the fourteen face shields were CE marked according to the stockpile database. Each face shield was removed from its packaging and assembled where applicable. The face shield's dimensions (Supplementary Figure SF2) were measured with a steel ruler or flexible tape measure, where appropriate, before mounting the face shield on a manikin head. There was a broad variation in the dimensions and design across the fourteen face shields e.g. the overall length of the face shield ranged $139-310 \mathrm{~mm}$ and the distance from the face shield to the ear ranged from 
an overlap of $25 \mathrm{~mm}$ to a gap of $70 \mathrm{~mm}$. Four of the face shields required self-assembly and ten had foam headbands.

Two manikin heads were used (Supplementary Figure SF3); representing male and female head forms from different global regions. The larger manikin head had a circumference of approximately $59 \mathrm{~cm}$ and the smaller head had a circumference of approximately $52 \mathrm{~cm}$.

Sampling templates were laser-cut from laboratory Cytiva Whatman Benchkote Surface Protector (Fisher Scientific UK Limited), an absorbent paper fibre-based material with a plastic backing, which had previously been shown to be non-UV fluorescent. The dimensions of both templates are shown in Supplementary figure SF4. Two templates, one for each manikin head size, were designed to capture deposit of fluorescein droplets on the face of the manikin head, the templates being delineated into three regions: eyes, nose, and mouth. The templates were pre-labelled according to the face shield model, manikin head size and position. The appropriately sized template was placed over the face of the manikin head, an example shown in Figure 2, and secured in place using adhesive tape over tabs at the sides of the eye and mouth sections.

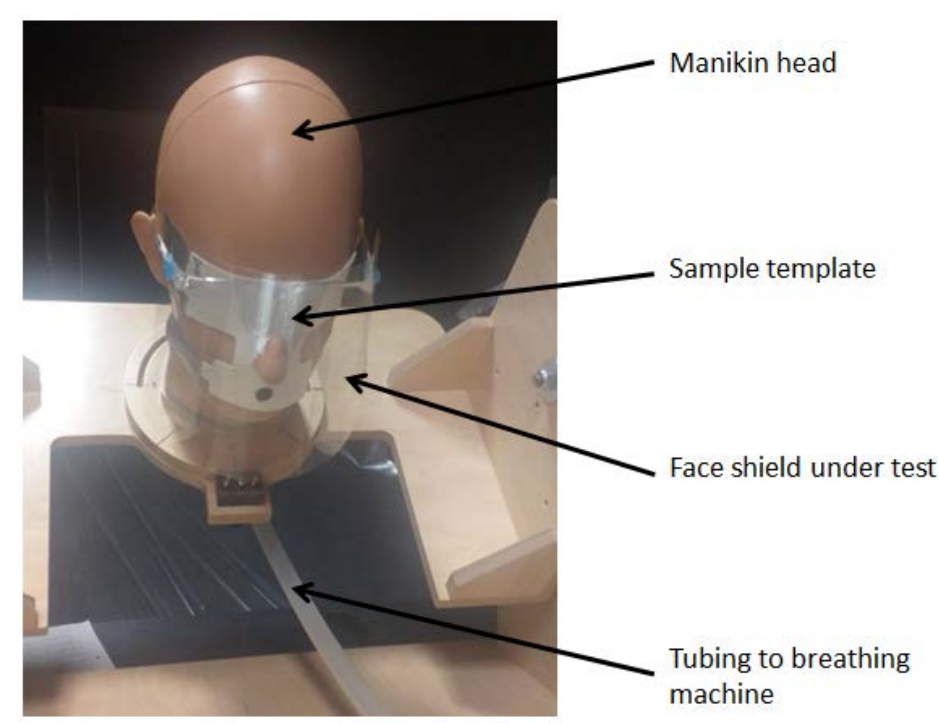

Figure 2. A sample template fixed to the manikin head whilst attached to the test rig. The manikin head is attached to the breathing machine via tubing at the neck.

An ultraviolet (UV) light (Titan365 UV LED, UV Light Technology, Birmingham, UK) was used to confirm that the sample was devoid of any fluorescence before proceeding. The face shield was then mounted on the head following the manufacturer's instructions, where provided and the manikin head was adjusted to the required position. The distance between the edge of the face shield and the base of the ear was measured on each side to determine the extent of coverage of the face. Any other observations were also noted.

The test set-up is shown in Figure 3. The cough simulator was positioned $60 \mathrm{~cm}$ in front of the manikin head and level with the eyes (Figure 3). Preliminary experiments delivering a cough to the manikin heads without face shields in place confirmed the even distribution of droplets across the templates. 


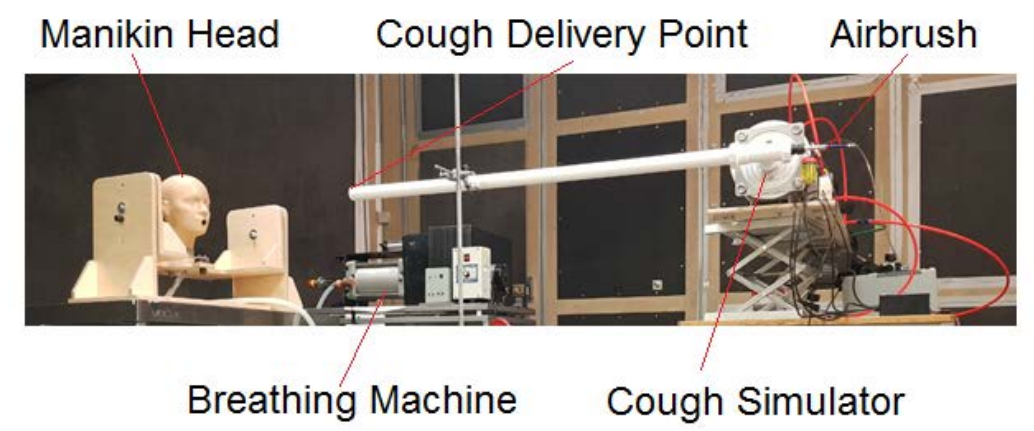

Figure 3. Cough simulator positioned in front of the manikin head rig and breathing machine to the side.

Each 'cough' was synchronised with the inhalation phase of the breathing cycle and repeated three times. After three consecutive 'coughs', the face shield was removed and the template was taken off the manikin head, handling only the tabs/tape to avoid cross-contamination of the deposition areas, then replaced with a new sample. Exposed samples were removed from the test area and stored in a clean space ready for further analysis.

Each test was conducted with the following manikin head positions, using a new absorbent material sample template but the same face shield, taking care not to cross contaminate the sample:

- Position 1: Facing forwards with the head face on.

- Position 2: Facing forwards and rotated 45 degrees backwards (front and looking up).

- Position 3: Facing forwards and rotated 45 degrees forwards (front and looking down).

- Position 4: Rotated 90 degrees to the left and rotated 45 degrees backwards (left and looking up).

- Position 5: Rotated 90 degrees to the left and rotated 45 degrees forwards (left and looking down).

- Position 6: Rotated 90 degrees to the right and rotated 45 degrees backwards (right and looking up).

- Position 7: Rotated 90 degrees to the right and rotated 45 degrees forwards (right and looking down).

Once all sample positions had been tested for each face shield, the room was then ventilated to remove any fine particles from the air.

The protocol was undertaken in triplicate with both the large and small manikin heads attached to the manikin rig and using each of the test face shields.

Four different models of goggles and one pair of safety glasses were also tested following the same method but with an adapted template (Supplementary Figure SF5).

A set of tests were undertaken to observe the ability of a face shield to contain a cough. The large manikin head on the test rig used in previous tests (Figure 2) was fitted with a standard template as shown in Supplementary figure SF4. For these tests, this head was referred to as the 'receiver'. The outlet of the cough simulator was then fitted to a second large manikin head at the cough delivery point as shown in Figure 4. This manikin head was referred to as the 'wearer'. The same template was fitted to the receiver with a larger cut out at the mouth to ensure that the cough simulator's outlet was unobstructed. The 'mouth' of the 'wearer' 
was positioned $60 \mathrm{~cm}$ in front of the 'receiver' level with the 'eyes'. Tests were run as before with three consecutive coughs but only using sample position one. Five representative face shields were tested.

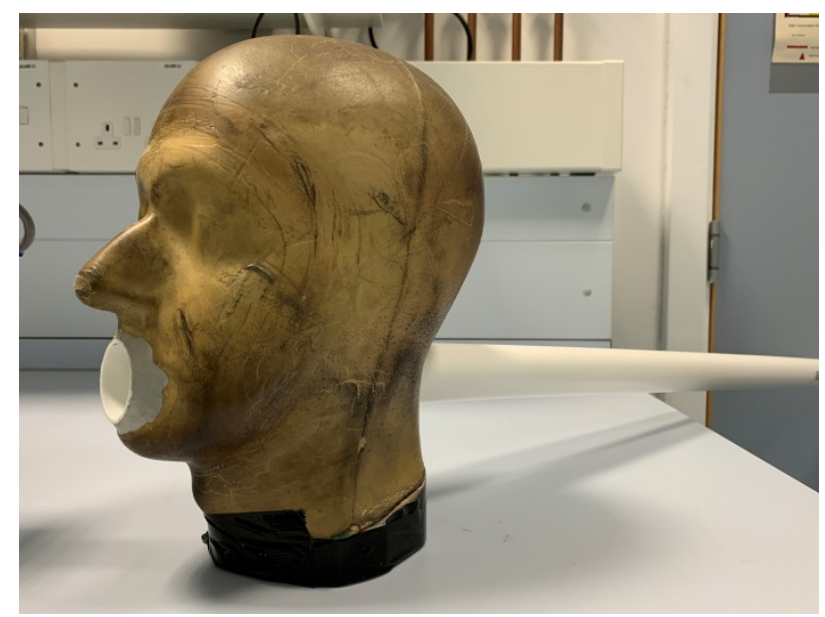

Figure 4. Cough simulator cough delivery point attached to the 'wearer' manikin head.

\section{Sample analysis}

Each sample was viewed under UV light and the presence or absence of fluorescent deposits in each region of the sample was separately recorded i.e. the eyes, nose and mouth.

This analysis weighted one single drop of fluorescence equally to if the area was totally contaminated.

It was considered useful to additionally classify the level of contamination in each area of the sample as 'Low', 'Medium' or 'High'. One researcher was assigned this task in order to reduce the variability of this subjective analysis. Examples of each of the three categories are shown in Supplementary Figure SF6. To aid analysis, each classification was also assigned a numerical score. Undetectable contamination $=0$, low $=1$, medium $=2$ and high $=3$.

\section{Results}

Tables $1-3$ show the mean levels of contamination in the eyes, nose and mouth regions when the manikin head was wearing each face shield. The mean levels of contamination in the eyes, nose and mouth regions of an exposed manikin head and a coughing manikin head when the coughing head was wearing five of the face shields is shown in Table 4. Mean levels of contamination in the eye region when wearing each of the four goggle types and one safety glasses type (G1 - G4 and SG1 respectively) is shown in Table 5. A description of each pair of goggles can be found in Supplementary Table ST1. 
Table 1. Face shields ranked best to worst based on the level of contamination in the eye region: lightest - low, to darkest - high, none (-). Head orientations: 1 - facing forwards, 2 - facing forwards looking up, 3 - facing forwards looking down, 4 - facing left looking up, 5 - facing left looking down, 6 - facing right looking up, 7 - facing right looking down.

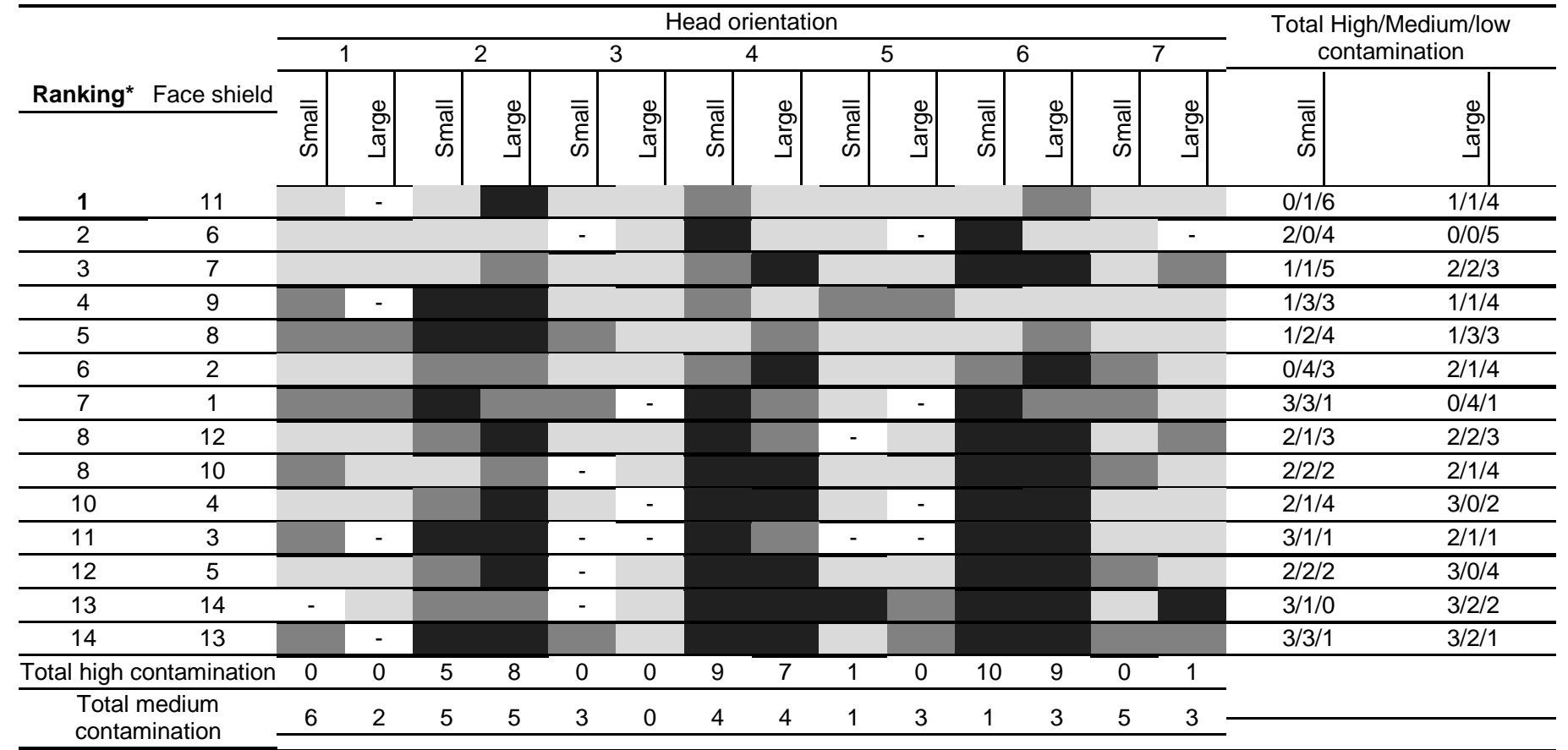

* Ranking criteria is the highest frequency of high level contamination, followed by the highest frequency of medium level contamination, followed by the highest frequency of low level contamination 
Table 2. Face shields ranked best to worst based on the mean level of contamination in the nose region: lightest - low, to darkest - high, none (-). Head orientations: 1 - facing forwards, 2 - facing forwards looking up, 3 - facing forwards looking down, 4 - facing left looking up, 5 - facing left looking down, 6 - facing right looking up, 7 - facing right looking down.

\begin{tabular}{|c|c|c|c|c|c|c|c|c|c|c|c|c|c|c|c|c|c|}
\hline \multirow[b]{3}{*}{ Ranking* } & \multirow[b]{3}{*}{ Face shield } & \multicolumn{14}{|c|}{ Head orientation } & \multirow{2}{*}{\multicolumn{2}{|c|}{$\begin{array}{c}\text { Total High/Medium/low } \\
\text { contamination }\end{array}$}} \\
\hline & & \multicolumn{2}{|c|}{1} & \multicolumn{2}{|c|}{2} & \multicolumn{2}{|c|}{3} & \multicolumn{2}{|c|}{4} & \multicolumn{2}{|c|}{5} & \multicolumn{2}{|c|}{6} & \multicolumn{2}{|c|}{7} & & \\
\hline & & $\begin{array}{l}\overline{\bar{\sigma}} \\
\text { फ }\end{array}$ & बై & $\begin{array}{l}\overline{\bar{\sigma}} \\
\stackrel{\bar{\varepsilon}}{\omega}\end{array}$ & ฮై & $\stackrel{\overline{\bar{\sigma}}}{\stackrel{E}{\omega}}$ & 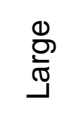 & $\begin{array}{l}\overline{\bar{\sigma}} \\
\stackrel{\bar{\omega}}{E}\end{array}$ & 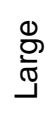 & $\begin{array}{l}\overline{\bar{\sigma}} \\
\stackrel{\mathcal{E}}{\omega}\end{array}$ & อิ & $\overline{\bar{\sigma}}$ & 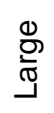 & $\overline{\bar{\sigma}}$ & 峁 & $\begin{array}{l}\overline{\bar{\sigma}} \\
\bar{\varepsilon}\end{array}$ & 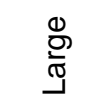 \\
\hline 1 & 6 & & & & & & & & & - & - & & 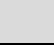 & & - & $1 / 1 / 4$ & $0 / 1 / 4$ \\
\hline 2 & 2 & & - & & & & & & & & & & & - & & $0 / 2 / 4$ & $1 / 1 / 4$ \\
\hline 3 & 11 & & & & & & - & & & & & - & - & - & & $0 / 1 / 4$ & $1 / 3 / 1$ \\
\hline 4 & 1 & & 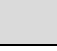 & & & & - & & & & & & & & & $1 / 3 / 3$ & $0 / 2 / 4$ \\
\hline 5 & 9 & & - & & & - & & & & & & & - & & - & $1 / 3 / 2$ & $1 / 0 / 3$ \\
\hline 6 & 8 & & . & & & & & & & & & & & & & $1 / 1 / 5$ & $1 / 2 / 4$ \\
\hline 7 & 4 & & - & & & & & & & & - & & & & & $0 / 3 / 4$ & $2 / 1 / 2$ \\
\hline 8 & 7 & & - & & & . & & & & & 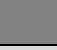 & & & & & $1 / 1 / 5$ & $1 / 4 / 1$ \\
\hline 9 & 10 & & & & & - & & & & & 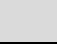 & & & & & $1 / 4 / 1$ & $1 / 2 / 4$ \\
\hline 10 & 3 & & & & & - & & & & & - & & & & - & $1 / 2 / 3$ & $2 / 1 / 2$ \\
\hline 11 & 5 & & & & & - & & & & - & & & & & & $1 / 3 / 1$ & $3 / 1 / 3$ \\
\hline 12 & 14 & - & . & & & . & & & & & & & & & & $2 / 2 / 2$ & $2 / 3 / 2$ \\
\hline 13 & 13 & - & 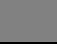 & & & - & - & & & & & & & & & $2 / 1 / 2$ & $3 / 3 / 0$ \\
\hline 14 & 12 & & - & & & & & & & & & & & & & $3 / 1 / 3$ & $3 / 2 / 1$ \\
\hline \multicolumn{2}{|c|}{ Total high contamination } & 0 & 0 & 7 & 8 & 0 & 0 & 1 & 6 & 0 & 0 & 7 & 7 & 0 & 0 & & \\
\hline \multicolumn{2}{|c|}{$\begin{array}{l}\text { Total medium } \\
\text { contamination }\end{array}$} & 1 & 3 & 5 & 4 & 0 & 0 & 10 & 6 & 4 & 5 & 4 & 3 & 4 & 5 & & \\
\hline
\end{tabular}

*Ranking criteria is the highest frequency of high level contamination, followed by the highest frequency of medium level contamination, followed by the highest frequency of low level contamination 
Table 3. Face shields ranked best to worst based on the mean level of contamination in the mouth region: lightest - low, to darkest - high, none (-). Head orientations: 1 - facing forwards, 2 - facing forwards looking up, 3 - facing forwards looking down, 4 - facing left looking up, 5 - facing left looking down, 6 - facing right looking up, 7 - facing right looking down.

\begin{tabular}{|c|c|c|c|c|c|c|c|c|c|c|c|c|c|c|c|c|c|}
\hline \multirow{3}{*}{ Ranking* } & \multirow{3}{*}{$\begin{array}{l}\text { Face } \\
\text { shield }\end{array}$} & \multicolumn{14}{|c|}{ Head orientation } & \multirow{2}{*}{\multicolumn{2}{|c|}{$\begin{array}{c}\text { Total High/Medium/low } \\
\text { contamination }\end{array}$}} \\
\hline & & \multicolumn{2}{|c|}{1} & \multicolumn{2}{|c|}{2} & \multicolumn{2}{|c|}{3} & \multicolumn{2}{|c|}{4} & \multicolumn{2}{|c|}{5} & \multicolumn{2}{|c|}{6} & \multicolumn{2}{|c|}{7} & & \\
\hline & & $\frac{\overline{\bar{\sigma}}}{\tilde{E}}$ & $\frac{\text { อ }}{\text { త్ర }}$ & $\begin{array}{c}\overline{\bar{\sigma}} \\
\overline{\tilde{\omega}}\end{array}$ & 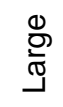 & $\frac{\overline{\bar{\sigma}}}{\stackrel{\omega}{\omega}}$ & ฮั & $\begin{array}{l}\overline{\bar{\sigma}} \\
\bar{\varepsilon}\end{array}$ & 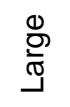 & $\frac{\overline{\bar{\sigma}}}{\sum^{\frac{1}{n}}}$ & $\frac{\mathscr{0}}{\text { ฮై }}$ & $\frac{\overline{\bar{\sigma}}}{\stackrel{\bar{\varepsilon}}{\omega}}$ & ฮั & $\overline{\overline{\bar{\sigma}}}$ & ฮั & $\begin{array}{l}\overline{\bar{\sigma}} \\
\text { ผ }\end{array}$ & 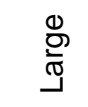 \\
\hline 1 & 6 & & - & & & & & & & & - & & - & & & $3 / 1 / 3$ & $0 / 1 / 3$ \\
\hline 2 & 11 & & & & & & & & & & & & & & & $2 / 2 / 3$ & $1 / 5 / 1$ \\
\hline 3 & 2 & & & & & - & & & & & & & & & & $1 / 5 / 0$ & $2 / 3 / 2$ \\
\hline 4 & 9 & & & & & & & & & & & & & & & $3 / 4 / 0$ & $1 / 3 / 3$ \\
\hline 5 & 3 & & & & & & & & & & & & & & & $3 / 3 / 1$ & $3 / 1 / 3$ \\
\hline 6 & 1 & & & & & & & & & & & & & & & $4 / 2 / 1$ & $2 / 3 / 2$ \\
\hline 7 & 4 & & - & & & & - & & & & & & & & & $4 / 1 / 2$ & $3 / 2 / 0$ \\
\hline 8 & 5 & & & & & & & & & & & & & & & $4 / 1 / 2$ & $3 / 2 / 2$ \\
\hline 9 & 7 & & & & & & & & & & & & & & & $2 / 5 / 0$ & $5 / 0 / 2$ \\
\hline 10 & 8 & & & & & & & & & & & & & & & $2 / 5 / 0$ & $5 / 2 / 0$ \\
\hline 11 & 12 & & & & & & & & & & & & & & & $4 / 1 / 2$ & $4 / 1 / 2$ \\
\hline 12 & 10 & & & & & & & & & & & & & & & $5 / 0 / 2$ & $4 / 1 / 2$ \\
\hline 13 & 13 & & & & & & & & & & & & & & & $5 / 0 / 2$ & $4 / 3 / 0$ \\
\hline 14 & 14 & & & & & - & & & & & & & & & & $5 / 0 / 1$ & $5 / 2 / 0$ \\
\hline \multicolumn{2}{|c|}{$\begin{array}{c}\text { Total high } \\
\text { contamination }\end{array}$} & 0 & 1 & 13 & 12 & 0 & 0 & 12 & 10 & 5 & 4 & 11 & 10 & 5 & 5 & & \\
\hline \multicolumn{2}{|c|}{$\begin{array}{l}\text { Total medium } \\
\text { contamination }\end{array}$} & 6 & 4 & 1 & 2 & 5 & 4 & 2 & 3 & 5 & 8 & 2 & 2 & 9 & 6 & & \\
\hline
\end{tabular}

* Ranking criteria is the highest frequency of high level contamination, followed by the highest frequency of medium level contamination, followed by the highest frequency of low level contamination 
Table 4. Mean level of contamination in the eye, nose and mouth regions when the coughing manikin head wore a face shield and the receiver did not: lightest - low, darkest - medium, none (-).

\begin{tabular}{|c|c|c|c|c|c|}
\hline \multirow{2}{*}{ Face shield } & \multicolumn{3}{|c|}{ Contamination on face of wearer } & \multicolumn{2}{c|}{ Deposition on face of receiver } \\
\cline { 2 - 6 } & Eyes & Nose & Mouth & Nose & \\
\hline 1 & & & & & \\
\hline 2 & & - & & & \\
\hline 6 & & & & & \\
\hline 10 & & & & & \\
\hline
\end{tabular}

Table 5. Goggles and safety glasses ranked best to worst based on the mean level of contamination in the eye region: lightest - low, to darkest - high. Head orientations: 1 - facing forwards, 2 - facing forwards looking up, 3 - facing forwards looking down, 4 - facing left looking up, 5 - facing left looking down, 6 - facing right looking up, 7 - facing right looking down.

\begin{tabular}{|c|c|c|c|c|c|c|c|c|c|c|c|c|c|c|c|c|}
\hline \multirow{3}{*}{ Ranking* } & \multirow{3}{*}{$\begin{array}{l}\text { Goggles/ } \\
\text { safety } \\
\text { glasses }\end{array}$} & \multicolumn{13}{|c|}{ Head orientation } & \multirow{2}{*}{\multicolumn{2}{|c|}{$\begin{array}{l}\text { Total High/Medium/low } \\
\text { contamination }\end{array}$}} \\
\hline & & \multicolumn{2}{|c|}{1} & \multicolumn{2}{|c|}{2} & \multicolumn{2}{|c|}{3} & \multicolumn{2}{|c|}{4} & \multicolumn{2}{|c|}{5} & \multicolumn{2}{|c|}{6} & 7 & & \\
\hline & & $\bar{\varepsilon} \overline{\bar{\sigma}}$ & ప & $\dot{\varepsilon} \overline{\bar{\sigma}}$ & ప & $\bar{\varphi} \overline{\bar{\sigma}}$ & ฮั ฮ & $\bar{\varepsilon} \overline{\bar{\sigma}}$ & สั ฮ & $\bar{\varphi} \overline{\bar{\sigma}}$ & ฮั ฮ & $\stackrel{\varepsilon}{\bar{\omega}} \overline{\bar{\sigma}}$ & ప & らૅ $\overline{\bar{\sigma}}$ & $\bar{\varepsilon} \overline{\bar{\sigma}}$ & ప ฮ \\
\hline 1 & G4 & & & & & & & & & & & & & & $0 / 4 / 3$ & $0 / 5 / 2$ \\
\hline 2 & G2 & & & & & & & & & & & & & . & $0 / 5 / 2$ & $0 / 5 / 2$ \\
\hline 3 & G1 & & & & & & & & & & & & & & $1 / 6 / 0$ & $0 / 6 / 1$ \\
\hline 4 & G3 & & & & & & & & & & & & & & $0 / 5 / 2$ & $2 / 5 / 0$ \\
\hline 5 & SG1 & & & & & & & & & & & & & & $5 / 1 / 1$ & $5 / 1 / 1$ \\
\hline $\begin{array}{r}\text { Total } \\
\text { Contaminatic } \\
\end{array}$ & $\begin{array}{l}\text { high } \\
\text { on - goggles }\end{array}$ & 0 & 0 & 0 & 0 & 0 & 0 & 0 & 1 & 0 & 0 & 1 & 1 & 0 & & \\
\hline $\begin{array}{r}\text { Total m } \\
\text { contaminatio }\end{array}$ & $\begin{array}{l}\text { edium } \\
\text { n - goggles }\end{array}$ & 4 & 4 & 3 & 4 & 1 & 4 & 3 & 2 & 4 & 2 & 2 & 2 & 3 & & \\
\hline $\begin{array}{r}\text { Total } \\
\text { Contaminati } \\
\text { glas }\end{array}$ & $\begin{array}{l}\text { high } \\
\text { on - safety } \\
\text { ses }\end{array}$ & 1 & 1 & 1 & 1 & 1 & 1 & 1 & 1 & 0 & 0 & 1 & 1 & 0 & & \\
\hline $\begin{array}{r}\text { Total } m \\
\text { contamir } \\
\text { safety g }\end{array}$ & $\begin{array}{l}\text { edium } \\
\text { hation - } \\
\text { lasses }\end{array}$ & 0 & 0 & 0 & 0 & 0 & 0 & 0 & 0 & 0 & 0 & 0 & 0 & 1 & & \\
\hline
\end{tabular}

* Ranking criteria is the highest frequency of high level contamination, followed by the highest frequency of medium level contamination, followed by the highest frequency of low level contamination_ 


\section{Discussion}

In this study, a wide variety of face shields were tested. While all offered some level of protection from a simulated cough challenge, none eliminated exposure. Tables 1 to 3 rank the face shields tested in order from best to worst for eyes, nose and mouth regions respectively. As may be expected, for both head sizes, the face shield offered the greatest overall protection when the manikin head was either face on (orientation 1) or looking down (orientation 3 front on; orientation 5 turned to the left and orientation 7 turned right). The face shield offered the least protection when the manikin heads were looking up (orientation 2 front on; orientation 4 turned to the left and orientation 6 turned to the right).Overall, the greatest challenge to the level of protection afforded by face shields was in the mouth region. In this region UV fluorescence deposition was highest, as depicted by the darkest grids, totalling 88 out of a possible $196(44.9 \%)$, compared to 50 at the eye region $(25.5 \%)$ and 36 at the nose region (18.4\%). This data supports the need for a further level of protection in the mouth region when working in close contact with patients and not undertaking aerosol generating procedures.

In summary, for any face region, face shields offer the greatest protection when a cough challenge is face on or from above (when the manikin head was tilted forward) and offer least protection when a cough challenge is from below, (when a manikin head is tilted backward). This has implications for situations where face shields are typically worn, for example in healthcare if the wearer is standing over an infected patient providing treatment, the angle of exposure is likely to come from below.

Across all three face regions (eyes, nose and mouth), the face shield designs that offered greatest protection against a cough challenge tended to be those with the most wrap around the face, i.e. the gap between the edge of the visor and the ear. For example, face shield 6 had a gap from visor edge to ear of $9 \mathrm{~mm}$ on the large head and $8 \mathrm{~mm}$ on the small head. With this face shield, only three instances of high level contamination were recorded in the mouth region with the small head and none with the large head. Conversely, the poorest performing face shields had wrap around less than many of the other face shields tested. For example, face shield 14 had a gap from visor edge to ear of $72 \mathrm{~mm}$ on the large head and $70 \mathrm{~mm}$ on the small head. With this face shield, five instances of high level contamination were recorded with both small and large head.

Large (width and length) visor areas were a feature of the two most protective face shields (6 and 11), while the worst performing ones in this study (13 and 14) both had small visor areas. In terms of wearer comfort, despite their greater level of protection, face shields 6 and 11 had cardboard headbands and straps which could make them more difficult to use than some other designs. Also, their close fit meant they either touched or pressed heavily against the nose on both manikin heads, suggesting discomfort during normal wear. As a result, it's possible that they would be tipped backward when worn and likely to afford less protection than was achieved during testing. The close fit may also make these models incompatible with some respirators that may need to be worn when undertaking aerosol generating procedures in healthcare. Of the poorly performing face shields, face shield 13 had a foam headband which is likely to be more comfortable. However, rather than an elastic strap it had two notched plastic straps that slotted together, which could be less secure. Face shield 14 had a plastic headband and thin rubber bands in place of a strap, with a noticeable gap between the headband and the forehead when worn on both manikin heads.

Greater protection was probably more influenced by the overall shaping of the visor. While it was assumed that the presence of a foam headband would provide greater protection, especially to the eyes and nose, this was not borne out as both best and worst performers had foam headbands. However, one of the poorer performers did have an obvious gap between the headband and the forehead.

With the cough simulator configured to deliver cough through a manikin head wearing five representative face shields (wearer), it was found that all those tested mitigated 
exposure to a manikin directly in front (receiver), with only one (face shield 10) allowing medium level deposition (Table 4). There were only two instances of self-contamination of the face inside the face shield. This suggests that wearing a face shield can provide some protection from cough droplets to those standing directly in front of the wearer. This was consistent with the findings from previous studies (Pan et al 2020 and Wendling et al 2021).

All the goggles tested allowed medium level exposure; even the best performing goggles (G4) allowed on average four medium level exposures across the seven orientations on the small head and five medium level exposures across the seven orientations on the large head (Table 5). Deposition was detected in every test; there were only seven instances where low deposition was detected across the 56 data points for the small head $(12.5 \%$ of the total number) and five out of 56 (8.9\%) for the large head.

As was the case with face shields, the manikin head orientations in which goggles provided greatest protection were with the head looking down (orientation 5; left looking down) on both head sizes with slightly greater protection on the large head; and orientation 7 (right looking down) on both size heads. Also consistent with face shields, orientations that were least protective were looking up; orientation 6 (right looking up) yielded two high level and two medium level depositions on each size head, as did orientation 4 (left looking up) on the large head. In general, the level of deposition on the face did not appear to correlate to the presence of vents in the goggle design, or gaps when fitted to the manikin heads. While the best performing goggles (G4) were observed to achieve a good seal around the forehead and cheeks which may have improved performance, they were found to be the least comfortable to wear due to lack of flexibility.

Safety glasses were much less protective than goggles (Table 5), allowing high level exposure in five out of seven orientations.

A key limitation of this study was that the authors weren't able to measure the size distribution of the droplets. It is believed that difficulties were due to the low concentration and relatively high velocity of the droplets being expelled from the cough simulator.

\section{Conclusions}

This study has contributed to the evaluation of face protection by developing methodology to simulate a human cough, and to visualise and provide a semi-quantitative assessment of droplet deposition. This semi-quantitative assessment has enabled a comparison to be made of the protection afforded by a range of face shield designs. It has also highlighted real world circumstances where the protection afforded by face shields may be compromised, for example with a cough challenge from below and angled up at the wearer. Previous studies have demonstrated protection with a face shield compared to without, but only used a single generic face shield (Lindsley et al 2014, Verma et al 2020 and Salimina et al 2021), or reviewed information on face shield design and performance, sometimes with illustrated examples (Roberge 2016 and Singh et al 2021) but without measured levels of protection. One study (Ko-Keeneya et al 2020) used fluorescence visualisation and looked at improvements afforded by amending a face shield design and addressed orientation, but again tested only a single style and limited orientations. Compared to previously published data, our study therefore offers a more comprehensive evaluation of face shield designs and protection afforded, as well as that from goggles and glasses.

The face shields, goggles and safety glasses reduced but did not eliminate the risk of exposure to the wearer from droplets such as those produced by a human cough. The level of protection differed based on the design of the eye protector and the relative orientation of the wearer to the cough e.g. face shields and goggles offered the greatest protection when a cough challenge was face on or from above and the least protection when a cough challenge was from below. Wearing a face shield can provide some protection from cough droplets to those standing directly in front of the wearer. Awareness of the advantages and limitations of different face shield designs can offer the user an informed choice as to 
how much protection they will provide, as part of a PPE ensemble, against airborne transmissible infection.

Data Availability: The data underlying this article will be shared on reasonable request to the corresponding author.

Acknowledgements: The authors would like to thank Amy Kalay for liaising with the NHS supply chain to source and supply the eye protection samples and Terry Gee for building the manikin head test rig and creating the absorbent material sample templates.

Funding: This publication and the work it describes were funded by the Health and Safety Executive (HSE). Its contents, including any opinions and/or conclusions expressed, are those of the authors alone and do not necessarily reflect HSE policy. The authors declare no conflict of interest relating to the material presented in this article.

\section{References}

Anon, JB, Denne, C, and Rees, D (2020) Patient-Worn Enhanced Protection Face Shield for Flexible Endoscopy. Otolaryngology - Head and Neck Surgery; 163:2 280-283

BEIS and DCMS - Department for Business, Energy \& Industrial Strategy and Department for Digital, Culture, Media \& Sport (2021) Working safely during coronavirus (COVID-19) [online]. GOV.UK [Viewed 09 August 2021] Available from: https://www.gov.uk/guidance/working-safely-during-covid-19/shops-branches-and-close-contact-services

BSI - British Standards Institution (2019) Medical face masks - Requirements and test methods BS EN 14683:2019

BSI - British Standards Institution (2009) Respiratory protective devices - Filtering half masks to protect against particles - Requirements, testing, marking BS EN 149:2001+A1:2009

BSI - British Standards Institution (2002a) Personal eye-protection - Specifications BS EN 166:2002

BSI - British Standards Institution (2002b) Personal eye-protection - Non-optical test methods BS EN 166:2002

Chu, DK, Akl, EA, Duda, S, Solo, K, Yaacoub, S and Schünemann HJ on behalf of the COVID-19 Systematic Urgent Review Group Effort (SURGE) study authors (2020) Physical distancing, face masks, and eye protection to prevent person-to-person transmission of SARS-CoV-2 and COVID-19: a systematic review and meta-analysis. Lancet; 395: 1973-87.

EU - European Union (2020) Commission Recommendation (EU) 2020/403 of 13 March 2020 on conformity assessment and market surveillance procedures within the context of the COVID-19 threat. Official Journal of the European Union: LI 79/1

Ko-Keeneya, EH, Sarana, MS, McLaughlinb, K and Lipmanc, S (2020) Improving protection from bioaerosol exposure during postoperative patient interaction in the COVID-19 era, a quality improvement study. Am J Otolaryngol; 41: 102634

Li, DTS, Samaranayake, LP, Leung, YY and Neelakantan, P (2020) Facial protection in the era of COVID-19: A narrative review. Oral Diseases; 27: (Suppl. 3) 665-673

Li, L, Niu, M and Zhu, Y (2021) Assessing the effectiveness of using various face coverings to mitigate the transport of airborne particles produced by coughing indoors. Aerosol Science and Technology; 55:3, 332-339

Lindsley, WG, Noti, JD, Blachere FM, Szalajda, JV and Beezhold DH (2014) Efficacy of Face Shields Against Cough Aerosol Droplets from a Cough Simulator. Journal of Occupational and Environmental Hygiene; 11: 509-518

Lindsley, WG, Reynolds, JS, Szalajda, JV, Noti, JD and Beezhold, DH (2013) A Cough Aerosol Simulator for the Study of Disease Transmission by Human Cough-Generated Aerosols. Aerosol Science and Technology; 47:8, 937-944

Lindsley, WG, Blachere, FM, Thewlis, RE, Vishnu, A, Davis, KA, et al (2010) Measurements of Airborne Influenza Virus in Aerosol Particles from Human Coughs. PLoS ONE; 5:11, e15100

MHRA - Medicines and Healthcare products Regulatory Agency (2021) [online] GOV.UK [Viewed 02 December 2021] Available from: https://www.gov.uk/guidance/regulatory-status-of-equipment-being-used-to-help-prevent-coronavirus-covid-19\#facemasks-and-face-coverings

Napoli, PE, Nioi, M, d'Aloja, E and Fossarello, M (2020) The Ocular Surface and the Coronavirus Disease 2019: Does a Dual 'Ocular Route' Exist? J. Clin. Med; 9: 1269

Pan, J, Harb, C Leng W and Marr, LC (2021) Inward and outward effectiveness of cloth masks, a surgical mask, and a face shield. Aerosol Science and Technology; 55:6, 718-733

Roberge, RJ (2016) Face shields for infection control: A review. Journal of Occupational and Environmental Hygiene; 13:4, 235-242

Samaranayakea, LP, Fakhruddinb, KS, Ngoc, HC, Changa, JWW and Panduwawala, C (2020) The effectiveness and efficacy of respiratory protective equipment (RPE) in dentistry and other health care settings: a systematic review. Acta Odontologica Scandinavica; 78:8, 626-639

Scalinci SZ, Battagliola, ET (2020) Conjunctivitis can be the only presenting sign and symptom of COVID-19. IDCases; 20 e00774 
Singh, P, Pal, K, Chakravraty, A and Ikram, S (2021) Execution and viable applications of face shield "a safeguard" against viral infections of cross-protection studies: A comprehensive review. Journal of Molecular Structure; 1238: 130443

Verma, S, Dhanak, M, and Frankenfield, J (2020) Visualizing droplet dispersal for face shields and masks with exhalation valves. Phys. Fluids; 32: 091701

Wendling, J-M, Fabacher, T, Pébaÿ, P-P, Cosperec, I, and Rochoy, M (2021) Experimental Efficacy of the Face Shield and the Mask against Emitted and Potentially Received Particles. Int. J. Environ. Res. Public Health; 18: 1942 